\title{
Application and Therapeutic Effect of Puncturing of the Costal Transverse Process for Pulsed Radiofrequency Treated TI-T3 Herpes Zoster Neuralgia
}

This article was published in the following Dove Press journal:

Journal of Pain Research

Jianjun Zhu ${ }^{1,2, *}$

Yong $\mathrm{Fei}^{2, *}$

Jiajia Deng ${ }^{2}$

Bin Huang ${ }^{2}$

Ming $\mathrm{YaO}^{2}$

'The Second Clinical Medical College, Zhejiang Chinese Medical University, Hangzhou, People's Republic of China; ${ }^{2}$ Department of Anesthesiology and Pain Research Center, The First Hospital of Jiaxing or The Affiliated Hospital of Jiaxing University, Jiaxing, People's Republic of China

*These authors contributed equally to this work
Correspondence: Ming Yao

Department of Anesthesiology and Pain Research Center, The First Hospital of Jiaxing or The Affiliated Hospital of Jiaxing University, No. 1882 South Zhonghuan

Road, Nanhu District, Jiaxing City,

Zhejiang Province, People's Republic of China

Tel +86573 I34562I8632

Email jxyaoming666@163.com
Purpose: In CT-guided dorsal root ganglion puncture, especially T1-T3, it is often difficult to reach the target due to obstruction of the lamina, transverse process, and ribs. Therefore, a safe and effective puncture method with high success rate is urgently needed to guide us in our clinical work.

Patients and Methods: A total of 44 patients with dorsal root ganglion underwent pulsed radiofrequency therapy for pain T1-T3 herpes zoster neuralgia at the pain department of Affiliated Hospital of Jiaxing University from January 2019 to February 2020 were retrospectively reviewed. Each patient underwent the same surgical method. The patient's operation time, CT filming times, nerve electrophysiological tests, the NRS scores before and after operation at one, four, eight, and 12 weeks, Pittsburgh Sleep Disorder Index (PSQI), dosage of gabapentin capsules and tramadol hydrochloride sustained-release tablets, surgical complications and incidence of postherpetic neuralgia (PHN) were recorded.

Results: The success rate of 44 patients who underwent puncturing of the costal transverse process to $\mathrm{T} 1$ target was $88.46 \%$, to T2 target was $90.68 \%$, and to T3 target was $90.68 \%$, respectively. The NRS score of patients before surgery was $5.48 \pm 0.59$, and those at one, four, eight, and 12 weeks after surgery were $3(3,3), 1(1,2), 0(0,1)$, and $0(0,0)$. The difference of NRS score between preoperation and postoperation is statistically significant. No intraoperative and postoperative complications occurred. Two patients developed PHN after standard treatment, and the incidence of it was $4.55 \%$.

Conclusion: CT-guided puncturing of the costal transverse process in the dorsal root ganglion of patients who underwent pulsed radiofrequency treatment of T1-T3 herpes zoster neuralgia showed a high success rate and is considered to be safe and effective.

Keywords: costotransverse joint, pulsed radiofrequency, dorsal root ganglion, herpes zoster neuralgia

\section{Introduction}

Herpes zoster infection occurs when varicella-zoster virus reactivates from the dorsal root ganglia or the brain nerves. This is more commonly seen in middle-aged and elderly individuals with reduced immunity. In the current treatment strategies for herpes zoster, the first is antiviral drugs, such as acyclovir, famciclovir, valaciclovir, etc. And the second is pain management, which mainly includes nonsteroidal anti-inflammatory drugs, opioids drugs, antiepileptic drugs and antidepressants; and then the next is nerve block, 
such as epidural block, paraspinal block and regional nerve block. The final is surgical treatment, such as pulsed radio frequency of dorsal root ganglion, radiofrequency thermocoagulation of dorsal root ganglion, electrical stimulation of spinal cord, morphine pump implantation, peripheral neurotomy, etc.

In terms of safety, compared with other treatments, surgical treatment has certain risks, such as local anesthetic poisoning, allergy, bleeding, infection, pneumothorax, spinal cord injury, etc. However, with the continuous improvement of the proficiency of clinical operation and the continuous standardization of the process, the safety of surgical treatment has also been continuously improved. In terms of effectiveness, the above treatments are effective in the acute stage of herpes zoster (disease duration $<1$ month), subacute stage (one month disease duration $<3$ months) or chronic phase (disease duration $>3$ months), but their effectiveness decreases obviously with the prolongation of the disease duration. ${ }^{1}$

Although there are several treatment strategies for treating herpes zoster, ${ }^{2}$ about $9-34 \%$ of these patients were associated with complications of herpes zoster neuralgia or postherpetic neuralgia (PHN) for months to even years. ${ }^{3,4}$ This in turn seriously affects the quality of life $^{5}$ and increases the medical burden of patients. ${ }^{5-8}$ Early effective intervention can shorten the duration as well as the severity of herpes zoster neuralgia, reducing the risk of $\mathrm{PHN}^{9}$ Recent clinical studies have revealed that pulsed radiofrequency treatment of dorsal root ganglion in managing herpes zoster neuralgia has achieved ideal therapeutic effects. ${ }^{10-12} \mathrm{CT}$-guided dorsal root ganglion puncture is considered safe and accurate, and so it is widely used for treating PHN clinically, ${ }^{13,14}$ However, it is worth mentioning that when herpes zoster is involved in the upper thoracic segment, especially in the area of dorsal root ganglion of T1-T3, CT-guided puncture often remains difficult due to blockage of the lamina, transverse process, and ribs. This not only prolongs the operation time, but also increases the patient's pain, making it difficult to obtain the expected operation results. So, in this study, a new method of puncturing the costal transverse process was designed to solve the difficulty of needle puncturing to reach the T1-T3 dorsal root ganglion.

\section{Patients and Methods}

\section{Clinical Data}

A total of 44 patients with herpes zoster neuralgia, including 19 males and 25 females, aged 42-85 years, with an average age of $(65.61 \pm 10.94)$ years, were selected at the Pain Department of the First Hospital of Jiaxing from
January 2019 to February 2020. All patients were given oral analgesics before undergoing treatment, and their pain still remained moderate to severe. The pulsed radiofrequency treatment was performed by the same doctor with experience in clinical pain management for all patients.

\section{Inclusion and Exclusion Criteria}

Inclusion criteria were as follows: the subjects met the clinical diagnostic criteria of herpes zoster neuralgia; the age of the subjects was $\geq 18$ years; the diseased region of the herpes zoster only involved the unilateral trunk nerve, that is, the thoracic 1-3 nerve; the subjects' pain numerical score (NRS) was higher than three when they were admitted to the hospital; during hospitalization, the subjects underwent pulsed radiofrequency modulation of the spinal dorsal root ganglion under the guidance of the CT scan (Subsidiary Table 2.); all the subjects agreed and received follow-up by telephone.

Exclusion criteria were as follows: patients with infections or tumors at the puncture site; who are allergic to drugs of recombinant human interferon $\alpha-2 b$, betamethasone, methyl cobalamin (Subsidiary Table 2), etc; with severe cardiovascular and cerebrovascular diseases, liver and kidney dysfunction, abnormal coagulation function; with poor blood sugar control; who are on long-term use of immunosuppressive agents or systemic failure; and with mental illness who cannot cooperate with surgery. This study was approved by the Ethics Committee of the First Hospital of Jiaxing (LS-2020-118) and conducted in accordance with the Declaration of Helsinki. Signed informed consent was obtained from all patients before surgery.

\section{Surgical Methods}

The patient was laid in a prone position for undergoing oxygen inhalation through the nasal duct and for monitoring of the vital signs (Subsidiary Table 2). The positioning grid was fixed on the back of the affected side with adhesive tape. The puncture route designed involves the following steps: the CT software tool ruler was used to draw a straight line from the ventral upper edge of the intervertebral foramen to the skin surface through a costotransverse joint, (Figure 1A, E and G). After routine disinfection and surgical towel spread, $1.0 \%$ lidocaine hydrochloride (Subsidiary Table 2) was used for local anesthesia infiltration. Under the guidance of CT, the radiofrequency trocar was slowly pushed forward. When the needle tip reaches the costotransverse joint (Figure 1B), then it was withdrawn slightly so that the needle point slope clung close to the head end of the costotransverse joint. The puncture needle 
(Subsidiary Table 2) was then pushed slowly, run down through the costotransverse joint (Figure 1C), and rotated by $180^{\circ}$. The puncture needle was slowly pushed and rotated by $90^{\circ}$ clockwise when the lamina was touched, making the needle point slope close to the lamina and slide slowly. The puncture needle was rotated by $180^{\circ}$ and slowly pushed forward so that the back tip of the puncture needle faced towards the outlet of the nerve root. Finally, the needle tip was located at the outlet of the nerve root at the ventral superior margin of the intervertebral foramen (Figure 1D F and H), and this could induce root pain from the back to the chest wall. The location of the puncture needle was verified by three-dimensional reconstruction (Figure 1I). The parameters of the sensory test were set at a voltage of $0.1-0.5 \mathrm{~V}$ and a frequency of $50 \mathrm{~Hz}$, and these could induce discomfort such as soreness, swelling, numbness or tingling in the original pain area. Low-frequency current was used in the exercise test, and the parameters were set at a voltage of $0.1-0.5 \mathrm{~V}$ and a frequency of $2 \mathrm{~Hz}$, which subsequently causes fibrillation and pulsation of the trunk muscle fibers in the corresponding segments. The temperature, time, pulse width and frequency were set at $42^{\circ} \mathrm{C}, 300$ seconds, $20 \mathrm{msec}$ and $2 \mathrm{~Hz}$, respectively. After the end of pulsed radiofrequency (Subsidiary Table 2), the electrode core was pulled out, and the puncture needle was withdrawn with no blood, gas, or liquid. Next, $5 \mathrm{~mL}$ of treatment solution was injected into each segment. The treatment solution was formulated with $100 \mathrm{mg}$ of $2 \%$ lidocaine hydrochloride, $1 \mathrm{mg}$ of mecobalamin injection, $1 \mathrm{mg}$ of compound betamethasone (Subsidiary Table 2), 1 million $\mathrm{U}$ of interferon $\alpha-2 b$ and $3 \mathrm{~mL}$ of $30 \%$ Iohexol (Subsidiary Table 2), and then diluted to $15 \mathrm{~mL}$ using $0.9 \%$ normal saline. The puncture point was compressed after the needle was pulled out. After observation for $15 \mathrm{~min}$, the patient was returned to the ward with normal vital signs.

\section{Observation Indexes}

The length of operation; the number of times of CT scans; the NRS scores before and after operation at one, four, eight, and 12 weeks; Pittsburgh Sleep Disorder Index (PSQI) score; the dosage of gabapentin capsules and tramadol hydrochloride sustained-release tablets; the surgical complications and the incidence of postherpetic neuralgia.

\section{Power of Study}

Based on the results of a reference ${ }^{1}$ which studied the total effective rate of pulsed radiofrequency therapy for patients in different degree of disease, combined with our patients' information, we estimated that the total effective rate of treatment was about $65 \%$. According to the results of this study, the total effective rate was $95.45 \%$. Moreover, the power of the study was estimated as $80 \%$, with $95 \%$ confidence interval and a two-sided type I error of 5\%. Therefore, the present study required 23 patients in each treatment arm. To compensate for patients who may have been lost to follow-up, 44 patients were reviewed. Among them, 26 patients were included in the $\mathrm{T} 1$ treatment arm, 43 patients in the $\mathrm{T} 2$ treatment arm and 43 patients in the $\mathrm{T} 3$ treatment arm.

\section{Statistical Analysis}

Statistical analysis was performed using SPSS v. 25.0 (Subsidiary Table 2). Shapiro-Wilk (SW) test was performed to analyze the normality of measurement data. The normal distribution of measurement data was represented as mean \pm SD. Comparison between groups was analyzed by two independent sample t tests. The non-normally distributed measurement data was represented by median (quartile spacing) and analyzed by Mann-Whitney $U$-test. While the categorical data was expressed in frequency and analyzed by Pearson's chisquared test or Fisher's exact tests, as the case may be. $P<0.05$ was considered to be statistically significant.

\section{Results}

\section{Analysis of Clinical Characteristics of Patients}

In this study, 44 patients with herpes zoster neuralgia at the Pain Department of Affiliated Hospital of Jiaxing University were enrolled. The clinical characteristics of subjects, such as age, sex, and NRS pain score are shown in Table 1. During the entire study, three patients were lost to follow-up, and the rate of lost interviews was $6.82 \%$.

\section{Analysis of Puncture Effect of Puncture of the Costal Transverse Process}

The puncturing method was successfully designed. Based on the summary of years of clinical work experience, the operation time of $\leq 60 \mathrm{~min}$, the number of CT scans of $\leq 10$ times, and $0.1-0.5 \mathrm{~V}$ after nerve electrophysiological testing obviously induced muscle tremors in the corresponding innervation area or numbness. These three points represented successful puncture design.

Among the 44 patients, 26 had puncturing at T1 through costal transverse process joint, 43 at the T2 target, and 43 at the T3 target (Subsidiary Table 1). After completing the puncture, nerve electrophysiological tests induced muscle tremor or numbness in the corresponding 

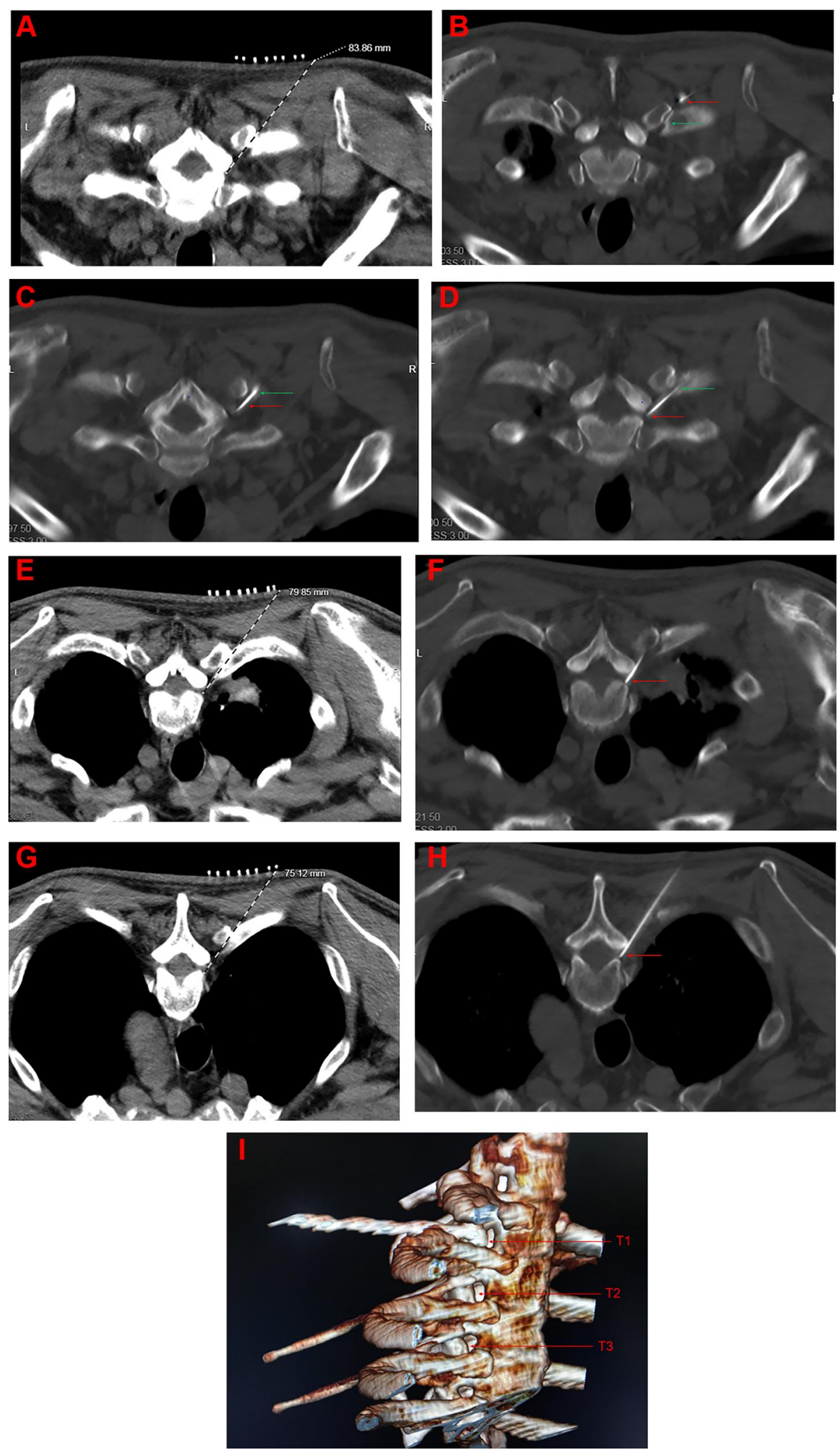

Figure I (A, E and G) CT-guided path design for TI, T2, and T3 puncture; (B) the tip of the puncture needle reached the TI costal transverse joint; (C) the puncture needle have successfully crossed the costal transverse joint; $(\mathbf{D}, \mathbf{F}$ and $\mathbf{H}) \mathbf{C T}$ images of TI, T2, and T3 puncture, finally and respectively; (I) CT three-dimensional reconstruction of TI, T2, T3 puncture.

Notes: The red arrow indicates the current position reached by the puncture needle, and the green arrow indicates the costal transverse process joint.

Abbreviations: TI, the intervertebral foramen of the first thoracic vertebra; T2, the intervertebral foramen of the second thoracic vertebra; T3, the intervertebral foramen of the third thoracic vertebra. 
Table I Clinical Characteristics of Patients

\begin{tabular}{|c|c|c|c|c|c|c|c|}
\hline \multirow[t]{2}{*}{ Patient No. } & \multicolumn{3}{|c|}{ Clinical Characteristics } & \multirow[t]{2}{*}{ Patient No. } & \multicolumn{3}{|c|}{ Clinical Characteristics } \\
\hline & Age & Sex & NRS & & Age & Sex & NRS \\
\hline I & 42 & Male & 6 & 23 & 66 & Male & 5 \\
\hline 2 & 46 & Female & 6 & 24 & 67 & Female & 5 \\
\hline 3 & 46 & Male & 5 & 25 & 68 & Female & 5 \\
\hline 4 & 49 & Female & 6 & 26 & 68 & Male & 5 \\
\hline 5 & 51 & Female & 5 & 27 & 70 & Male & 6 \\
\hline 6 & 52 & Female & 6 & 28 & 70 & Female & 6 \\
\hline 7 & 54 & Female & 5 & 29 & 71 & Male & 6 \\
\hline 8 & 54 & Female & 5 & 30 & 71 & Female & 4 \\
\hline 9 & 56 & Male & 5 & 31 & 72 & Male & 6 \\
\hline 10 & 57 & Female & 6 & 32 & 72 & Female & 5 \\
\hline II & 58 & Male & 6 & 33 & 73 & Male & 6 \\
\hline 12 & 58 & Female & 6 & 34 & 74 & Male & 5 \\
\hline 13 & 59 & Female & 5 & 35 & 74 & Female & 6 \\
\hline 14 & 59 & Male & 6 & 36 & 76 & Female & 6 \\
\hline 15 & 61 & Female & 5 & 37 & 76 & Male & 5 \\
\hline 16 & 63 & Female & 6 & 38 & 77 & Male & 5 \\
\hline 17 & 63 & Female & 6 & 39 & 77 & Female & 6 \\
\hline 18 & 63 & Female & 6 & 40 & 82 & Male & 5 \\
\hline 19 & 63 & Female & 6 & 41 & 83 & Male & 7 \\
\hline 20 & 64 & Female & 5 & 42 & 83 & Male & 5 \\
\hline 21 & 65 & Female & 5 & 43 & 84 & Male & 5 \\
\hline 22 & 65 & Female & 5 & 44 & 85 & Male & 5 \\
\hline
\end{tabular}

Abbreviation: NRS, numerical rating scale.

innervation area. Statistical analysis revealed that the rate of success of puncturing through $\mathrm{T} 1$ costal transverse process joint was $88.46 \%$, the success rate of puncturing through T2 costal transverse process joint was $90.48 \%$, and the success rate of puncture through T3 costal transverse process joint was $90.48 \%$.

\section{Treatment Effect Analysis NRS Score and PSQI Score}

The NRS scores of the patients before the operation were evaluated and a three-month telephone follow-up was conducted. The NRS scores of the patients were recorded at one, four, eight, and 12 weeks after the operation (Table 2). At the same time, the PSQI scores of patients before and after surgery were evaluated. The preoperative PSQI score was $9.67 \pm 1.60$, while the postoperative PSQI score was $2.77 \pm 0.97, P=0.014$, respectively, and the difference was statistically significant.

\section{Intraoperative and Postoperative Complications}

Based on several years of clinical work experience, the common complications associated with puncturing of costal transverse process are mainly pneumothorax, nerve injury, local anesthetic poisoning, and even the whole spinal anesthesia. During this experiment, none of the patients experienced the above complications.

\section{Postoperative Drug Treatment}

Statistical analysis was conducted on the dosages of gabapentin capsules and tramadol hydrochloride sustainedrelease tablets before and after surgery in patients. The dosages of gabapentin capsules and tramadol hydrochloride sustained-release tablets before and after surgery showed statistically significant differences (Table 3).

\section{PHN Incidence Analysis}

During telephonic follow-up, two patients had neuralgia in the corresponding area before surgery and three months after treatment. The PHN of T1-T3 herpes zoster neuralgia after puncturing of costal transverse process at the dorsal root ganglion was $4.55 \%$. Radiofrequency ablation of the corresponding segment of the dorsal root ganglion was performed in two patients, and the results were good, showing significantly reduced postoperative pain. 


\section{Discussion}

Herpes zoster neuralgia is a relatively common disease clinically, ${ }^{8}$ but its mechanism remains complex, and treatment remains difficult, ${ }^{15}$ and so is also known as the "undead cancer". Herpes zoster neuralgia mainly occurs due to the activation of the varicella-zoster virus that lurks the dorsal root ganglion or brain ganglion, causing damage to the nerve axons. This in turn damages the sensory nerves and produces abnormally charged impulses, transmitting pain to the spinal cord. The dorsal root ganglion is the first-stage neuron that transmits the impulses from the peripheral nerve to the spinal cord and then to the upper center. Therefore, blocking the spinal ganglion can block the transmission of peripheral pain signals to the upper center, thereby inhibiting the release of excitatory transmitters and regulating changes in central plasticity. ${ }^{16,17}$

In clinic, pulsed radiofrequency (PRF) of dorsal root ganglion (DRG) has been widely used in many diseases, such as knee osteoarthritis, ${ }^{18,19}$ pubic neuralgia, ${ }^{20}$ postherpetic neuralgia, ${ }^{21}$ occipital neuralgia, ${ }^{21}$ glossopharyngeal neuralgia, ${ }^{22}$ etc, due to its advantages of less destructiveness, quick recovery, and obvious effect. ${ }^{23}$

In recent years, the research on the mechanism of PRF has been carried out gradually. In the SNI rat model, it was found that PRF could relieve neuropathic pain by inhibiting the overexpression of IRF8 and BDNF in the spinal cord of $\mathrm{NAC}^{24}$ and inhibiting the activation of spinal microglia to downregulate DNF and the phosphorylation of PI3K and ERK. ${ }^{25}$

While in the CCI rat model, it was found that PRF can upregulate the transcription and translation of GDNF in the compressed sciatic nerve, ${ }^{26}$ inhibit the activity of IRF8, p38 and microglia in the spinal cord of rats, ${ }^{27}$ downregulate the expression of chain proteins, ${ }^{28}$ and enhance the levels of interleukin-1 (IL-1) and tumor necrosis factor (TNF) in the blood of rats, ${ }^{28}$ and then achieve the purpose of relieving neuropathic pain.

Some researchers found that after PRF, the ultrastructure of neuronal axons was damaged, the membrane and mitochondria were abnormal, and the microtubules and microfilaments had collapsed under electron microscope.${ }^{28,29}$ It was also found that the expression of $\mathrm{Na} / \mathrm{Katp}$ enzyme and c-Fos in spinal cord was upregulated after pulsed radiofrequency treatment of dorsal root ganglion, ${ }^{30}$ and increased the change of synaptic transmission. ${ }^{23}$ Thus, it can be seen that PRF can regulate the synaptic plasticity of nerve cells, ${ }^{23}$ and it is speculated that the analgesic effect of PRF may be realized by neuroregulation.

CT-guided dorsal root ganglion puncture is considered safe and accurate, but the distribution of dorsal root ganglion in the upper thoracic segment, especially $\mathrm{T} 1-\mathrm{T} 3$, often remains difficult to reach the target due to obstruction of the lamina, transverse process, and ribs. In view of the difficulty in puncturing of the dorsal root ganglion of T1-T3, our study designed thoracic sympathetic nerve radiofrequency technology and improved it, making it easier for the CT-guided puncturing of the costal transverse process to reach to the target. Our research team has previously studied CT-guided thoracic sympathetic nerve radiofrequency technology (T4) for treating Raynaud's syndrome or hand sweat and achieved satisfactory results. ${ }^{31}$ CT-guided thoracic sympathetic nerve radiofrequency puncture path was designed as follows: the CT's own software tool was used to draw a straight line from the outside of the ribs to the wall of the pleura through transverse joint of the ribs to the skin surface. After that, the CT-guided puncture needle passes the costal transverse process joint, rotates the puncture needle to $180^{\circ}$, and slowly advances the puncture needle against the rib head and the wall pleura. Finally, the tip of the puncture needle was located outside the ribs and close to the pleural wall. The puncturing of T1-T3 dorsal root ganglion is similar to the radiofrequency puncturing of thoracic sympathetic nerve. However, after the puncture needle crosses the costotransverse joint, the direction of the puncture needle should be adjusted to $1 / 3$ of the intervertebral foramen, and the operation can be completed after crossing and advancing into the lamina.

Based on many years of clinical work, the main complications of such puncture techniques include pneumothorax, nerve injury, local anesthetic poisoning, and even whole spinal anesthesia. This study mainly prevented the above complications by standardizing the puncturing procedure. This involves the determination of puncture site under CT positioning, and the puncture action should not be too strong. After the puncture needle crosses the rib transverse joint, it slowly advances to avoid the occurrence of complications due to violence. Finally, the drug should be withdrawn before administration and make sure it is administered without bleeding. When the above complications occur, then the operation should be immediately stopped and symptomatic treatment should be given.

Of the 44 patients in this study, 26 patients underwent puncturing to the $\mathrm{T} 1$ target through transcostal joint process, in which 23 were considered successful treatment; 43 
Table 2 Comparison of Preoperative and Postoperative NRS

\begin{tabular}{|c|c|c|c|c|c|}
\hline & \multirow[t]{2}{*}{ Before Operation } & \multicolumn{4}{|c|}{ After Operation } \\
\hline & & I Week & 4 Weeks & 8 Weeks & 12 Weeks \\
\hline \multicolumn{6}{|c|}{ Mann-Whitney U-test } \\
\hline$g^{2}$ & 0.50 & 8.18 & 7.30 & 18.04 & 23.76 \\
\hline$\alpha$ & 0.13 & 4.80 & 5.27 & 9.46 & 11.39 \\
\hline NRS & $5.48 \pm 0.59$ & $3(3,3)$ & $I(I, 2)$ & $0(0,1)$ & $0(0,0)$ \\
\hline$P$ & & $0.00 I^{*}$ & $0.00 I^{*}$ & $0.00 I^{*}$ & $0.00 I^{*}$ \\
\hline
\end{tabular}

Note: $* p<0.001$.

Abbreviations: $\mathrm{g}^{2}$, the kurtosis coefficient; $\alpha$, the skewness coefficient.

Table 3 Comparison of Preoperative and Postoperative Medication

\begin{tabular}{|c|c|c|}
\hline & Before Operation & After Operation \\
\hline \multicolumn{3}{|c|}{ Gabapentin } \\
\hline \multicolumn{3}{|c|}{ Mann-Whitney U-test } \\
\hline$g^{2}$ & 0.38 & 0.23 \\
\hline$\alpha$ & 0.56 & 0.01 \\
\hline$P$ & & $0.002^{*}$ \\
\hline \multicolumn{3}{|c|}{ Tramadol hydrochloride } \\
\hline \multicolumn{3}{|c|}{ Mann-Whitney U-test } \\
\hline$g^{2}$ & 0.65 & 2.72 \\
\hline$\alpha$ & 13.55 & 8.19 \\
\hline$P$ & & $0.001 * *$ \\
\hline
\end{tabular}

Notes: $* P<0.01, * * p<0.001$

Abbreviations: $g^{2}$, the kurtosis coefficient; $\alpha$, the skewness coefficient.

to the T2 target, wherein 39 had successful treatment; and 43 to the T3 target. Postoperative NRS scores, PSQI scores, and dosages of gabapentin capsules and tramadol hydrochloride sustained-release tablets were significantly lower than before surgery. No surgical complications occurred in patients during and after surgery. According to the results of the three-month telephone follow-up, three patients were confirmed to have PHN. The postoperative PHN incidence rate is $4.55 \%$, and this is similar to the previous reports both at home and abroad. ${ }^{10}$

In summary, CT-guided puncture of the costal transverse process in the dorsal root ganglion with pulsed radiofrequency treatment of T1-T3 herpes zoster neuralgia demonstrated a high success rate and is considered to be safe and effective. This study provides a new technique guide for CT-guided puncture technology and also provides an effective reference for clinical treatment of patients with herpes zoster involving T1-T3 areas.

\section{Conclusion}

CT-guided puncturing of the costal transverse process in the dorsal root ganglion that underwent pulsed radiofrequency treatment of T1-T3 herpes zoster neuralgia demonstrated a high success rate, and is considered to be safe and effective.

\section{Acknowledgments}

The authors thank the subjects of this study for participating in this study. This work was supported by funding from Zhejiang provincial and municipal construction plan for key disciplines (2019-ss-ttyx), Jiaxing Key Laboratory of Neurology and Pain Medicine and Jiaxing Science and Technology Bureau (2019AD32150).

\section{Disclosure}

The authors declare no conflicts of interest in this work. 


\section{References}

1. Ding Y, Li H, Hong T, Zhao R, Yao P, Zhao G. Efficacy and safety of computed tomography-guided pulsed radiofrequency modulation of thoracic dorsal root ganglion on herpes zoster neuralgia. Neuromodulation. 2019;22(1):108-114. doi:10.1111/ner.12858

2. Gross GE, Eisert L, Doerr HW, et al. S2k guidelines for the diagnosis and treatment of herpes zoster and postherpetic neuralgia. $J$ Dtsch Dermatol Ges. 2020;18(1):55-78. doi:10.1111/ddg.14013

3. Shrestha M, Chen A. Modalities in managing postherpetic neuralgia. Korean J Pain. 2018;31(4):235-243. doi:10.3344/kjp.2018.31.4.235

4. Kramer S, Baeumler P, Geber C, et al. Somatosensory profiles in acute herpes zoster and predictors of postherpetic neuralgia. Pain. 2019;160(4):882-894. doi:10.1097/j.pain.0000000000001467

5. Saguil A, Kane S, Mercado M, Lauters R. Herpes zoster and postherpetic neuralgia: prevention and management. Am Fam Physician. 2017;96(10):656-663.

6. Meyers JL, Candrilli SD, Rausch DA, Yan S, Patterson BJ, Levin MJ. Costs of herpes zoster complications in older adults: A cohort study of US claims database. Vaccine. 2019;37(9):1235-1244. doi:10.1016/ j.vaccine.2018.11.079

7. Mareque M, Oyaguez I, Morano R, Casado MA. Systematic review of the evidence on the epidemiology of herpes zoster: incidence in the general population and specific subpopulations in Spain. Public Health. 2019;167:136-146. doi:10.1016/j.puhe.2018.10.015

8. Matthews I, Duong M, Parsons VL, et al. Burden of disease from shingles and post-herpetic neuralgia in the over 80 year olds in the UK. PLoS One. 2020;15(2):e0229224. doi:10.1371/journal. pone.0229-224

9. Rullan M, Bulilete O, Leiva A, et al. Efficacy of gabapentin for prevention of postherpetic neuralgia: study protocol for a randomized controlled clinical trial. Trials. 2017;18(1):24. doi:10.1186/s13063016-1729-y

10. Esposito MF, Malayil R, Hanes M, Deer T. Unique characteristics of the dorsal root ganglion as a target for neuromodulation. Pain Med. 2019;20(Suppl 1):S23-S30. doi:10.1093/pm/pnz012

11. Kim ED, Lee YI, Park HJ. Comparison of efficacy of continuous epidural block and pulsed radiofrequency to the dorsal root ganglion for management of pain persisting beyond the acute phase of herpes zoster. PLoS One. 2017;12(8):e0183559. doi:10.1371/journal. pone. 0183559

12. Kim K, Jo D, Kim E. Pulsed radiofrequency to the dorsal root ganglion in acute herpes zoster and postherpetic neuralgia. Pain Physician. 2017;20(3):E411-E418. doi:10.36076/ppj.2017.E418

13. Jia Y, Chen Z, Ren H, Luo F. The effectiveness and safety of 42 degrees $\mathrm{C}$ pulsed radiofrequency combined with 60 degrees $\mathrm{C}$ continuous radiofrequency for refractory infraorbital neuralgia: a prospective study. Pain Physician. 2019;22(3):E171-E179.

14. Lazzari ZT, Palmisani S, Hill B, Al-Kaisy A, Lambru G. A prospective case series of sphenopalatine ganglion pulsed radiofrequency therapy for refractory chronic cluster headache. Eur J Neurol. 2020;27(7):1190-1196. doi:10.1111/ene. 14176

15. Gan EY, Tian EA, Tey HL. Management of herpes zoster and postherpetic neuralgia. Am $J$ Clin Dermatol. 2013;14(2):77-85. doi:10.1007/s40257-013-0011-2

16. Lin CS, Lin YC, Lao HC, Chen CC. Interventional Treatments for postherpetic neuralgia: a systematic review. Pain Physician. 2019;22 (3):209-228.

17. Gierthmuhlen J, Braig O, Rehm S, Hellriegel J, Binder A, Baron R. Dynamic of the somatosensory system in postherpetic neuralgia. Pain Rep. 2018;3(6):e668. doi:10.1097/PR9.0000000000000668
18. Gulec E, Ozbek H, Pektas S, Isik G. Bipolar versus unipolar intraarticular pulsed radiofrequency thermocoagulation in chronic knee pain treatment: a prospective randomized trial. Pain Physician. 2017;20 (3):197-206. doi:10.36076/ppj.2017.206

19. Gupta A, Huettner DP, Dukewich M. Comparative effectiveness review of cooled versus pulsed radiofrequency ablation for the treatment of knee osteoarthritis: a systematic review. Pain Physician. 2017;20(3):155-171. doi:10.36076/ppj.2017.171

20. Fang H, Zhang J, Yang Y, Ye L, Wang X. Clinical effect and safety of pulsed radiofrequency treatment for pudendal neuralgia: a prospective, randomized controlled clinical trial. J Pain Res. 2018;11:23672374. doi: $10.2147 /$ JPR.S167866

21. Chang MC. Efficacy of pulsed radiofrequency stimulation in patients with peripheral neuropathic pain: a narrative review. Pain Physician. 2018;21(3):E225-E234. doi:10.36076/ppj.2018.3.E225

22. Bharti N, Chattopadhyay S, Singla N, Bala I, Batra YK, Bakshi J. Pulsed radiofrequency ablation for the treatment of glossopharyngeal neuralgia secondary to oropharyngeal carcinoma. Pain Physician. 2018;21(3):295-302. doi:10.36076/ppj.2018.3.295

23. Cahana A, Vutskits L, Muller D. Acute differential modulation of synaptic transmission and cell survival during exposure to pulsed and continuous radiofrequency energy. J Pain. 2003;4(4):197-202. doi:10.1016/s1526-5900(03)00554-6

24. Fang X, Xu X, Lin X, Liu R. Downregulated spinal IRF8 and BDNF in NAC are involved in neuropathic pain-induced depression relief via pulsed radiofrequency on dorsal root ganglion in rat SNI model. Brain Res Bull. 2019;146:192-200. doi:10.1016/j. brainresbull.2019.01.008

25. Xu X, Fu S, Shi X, Liu R. Microglial BDNF, PI3K, and p-ERK in the spinal cord are suppressed by pulsed radiofrequency on dorsal root ganglion to ease SNI-induced neuropathic pain in rats. Pain Res Manag. 2019;2019:5948686. doi:10.1155/2019/5948686

26. Hailong J, Hao R, Zipu J, Nan J, Fang L. Pulsed radiofrequency improves neuropathic pain in chronic constriction injury rats through the upregulation of the transcription and translation levels of glial cell line-derived neurotrophic factor. Pain Physician. 2018;21(1):33-40.

27. Liu R, Xu X, Xu Y, Fang X, Lin X. Pulsed radiofrequency on dorsal root ganglion relieved neuropathic pain associated with downregulation of the spinal interferon regulatory factor 8 , microglia, p38MAPK expression in a CCI rat model. Pain Physician. 2018;21(4):E307E322. doi:10.36076/ppj.2018.4.E307

28. Jiang R, Li P, Yao YX, et al. Pulsed radiofrequency to the dorsal root ganglion or the sciatic nerve reduces neuropathic pain behavior, decreases peripheral pro-inflammatory cytokines and spinal betacatenin in chronic constriction injury rats. Reg Anesth Pain Med. 2019;44(7):742-746. doi:10.1136/rapm-2018-100032

29. Erdine S, Bilir A, Cosman ER, Cosman. ER Jr. Ultrastructural changes in axons following exposure to pulsed radiofrequency fields. Pain Pract. 2009;9(6):407-417. doi:10.1111/j.15332500.2009.00317.x

30. Vallejo R, Tilley DM, Williams J, Labak S, Aliaga L, Benyamin RM. Pulsed radiofrequency modulates pain regulatory gene expression along the nociceptive pathway. Pain Physician. 2013;16(5):E601E613.

31. Huang H, Qiu W, Chen Q, Sun K, Huang B. Computed tomography (CT)-guided percutaneous thoracic sympathetic chain radiofrequency thermocoagulation for raynaud disease. Med Sci Monit. 2019;25:7391-7395. doi:10.12659/MSM.917392 


\section{Publish your work in this journal}

The Journal of Pain Research is an international, peer reviewed, open access, online journal that welcomes laboratory and clinical findings in the fields of pain research and the prevention and management of pain. Original research, reviews, symposium reports, hypothesis formation and commentaries are all considered for publication. The manuscript management system is completely online and includes a very quick and fair peer-review system, which is all easy to use. Visit http:// www.dovepress.com/testimonials.php to read real quotes from published authors. 